\title{
An object-oriented assembly modeling approach
}

\author{
Y. Yue ${ }^{1)}$, O. Salomons, H.J.J. Kals, F.J.A.M. van Houten \\ 1) Mechanical Engineering Department, Changsha Railway \\ University, P.R China, 410075 \\ e-mail: yangyue@csru.edu.cn \\ 2) Department of Mechanical Engineering, University of Twente \\ P.O. Box 217, 7500 AE Enschede, The Netherlands
}

\begin{abstract}
In order to solve the complexity of assembly modeling and CAD system development for some mechanical devices, which are mainly made up of components with standard structures, and to increase the design automation of components' local structure related to assembly, a system architecture for objectoriented assembly modeling and design is presented. Next, every component in machine is considered as an object, the basic component class and its expressions are founded. Based on this, a double layered and bi-directional chained list and its structure expressions are presented to represent linking relations in a component group and the ones among component groups are set up, and a basic component group class is set up. Furthermore, the approach to describe the mutual constraint relations between components is presented, so that the components' local details related to assembly can be designed automatically.
\end{abstract}

\section{Keywords}

assembly modeling, object-oriented method, computer aided design

\section{INTRODUCTION}

Assembly modeling is the core of machinery CAD system, and it has also great influence on the availability of the CAD system. Generally, there are two kinds of methods in assembly modeling, one is from bottom to top, and another is from top to bottom. The former is that the designers first carry out detailed structure designs to the components in a machine by CAD tool after whole design scheme is determined, then assembling relations of those components are defined and a product model is established. The latter is that function expressions of a product 
are built first, afterwards designers detail the geometry structures of components by a CAD system and assure that the structures of components are satisfied for the function requirements, then product model is built up.

The studies in bottom-up assembly modeling were made much in 1980's [Lee K et al.,1985],[Rocheleau D N et al.,1987], and [Shah J J, et al.,1989]. At the beginning of 1990's, Mäntylä M.A put forward the top-down assembly modeling thought [Mäntylä M.A, 1990], and described assembly models by means of Part-of Graphs consisting of nodes which stand for design features, and arcs which stand for the relations between nodes.

The mixture of these two methods is also recommended in computer aided assembly modeling [Sodhi $\mathrm{R}$ et al.,1994]. Both function requirements and design scheme constraint the structure designs of components, and the detailed structure of components affects reversibly the assembly design, hence component modeling and assembling modeling should be integrated.

Some machines, or mechanical devices like dies, consist mainly of subassemblies and components with standard structures, and local structures of these components will be modified when they are used to set up a new machine or device. In order to lower the complexity of assembly modeling and increase design automation to this kind of mechanical devices, it is necessary to adopt the objectoriented method in order to build up an effective CAD system related to practical design problem. Below we try to explain such an object-oriented assembly modeling approach.

\section{GENERAL REPRESENTATION OF ASSEMBLY MODELING}

Supposing a machine, or a mechanical device, consists of dozens of component groups (or sub-assemblies), and a component group consists of one or more components, we use $\mathrm{M}$ standing for the machine, Gi standing for a component group (or a subassembly), and Cij standing for a component, then $\mathrm{M}$ and $\mathrm{Gi}$ can be described as:

$M=\{G 1, G 2, \cdots \cdots, G i, \cdots \cdots\}$

$G i=\left\{C i 1, C i 2, \cdots \cdots, C_{i j}, \cdots \cdots.\right\}$

Any component in an assembly model can by described by a group of attributes: $C_{i j}=\{A 1, A 2, A 3\}$

In the above formulation, A1 stands for a component model, which including geometry and non-geometry information, and component position $(x, y, z, \alpha, \beta, \gamma)$, 


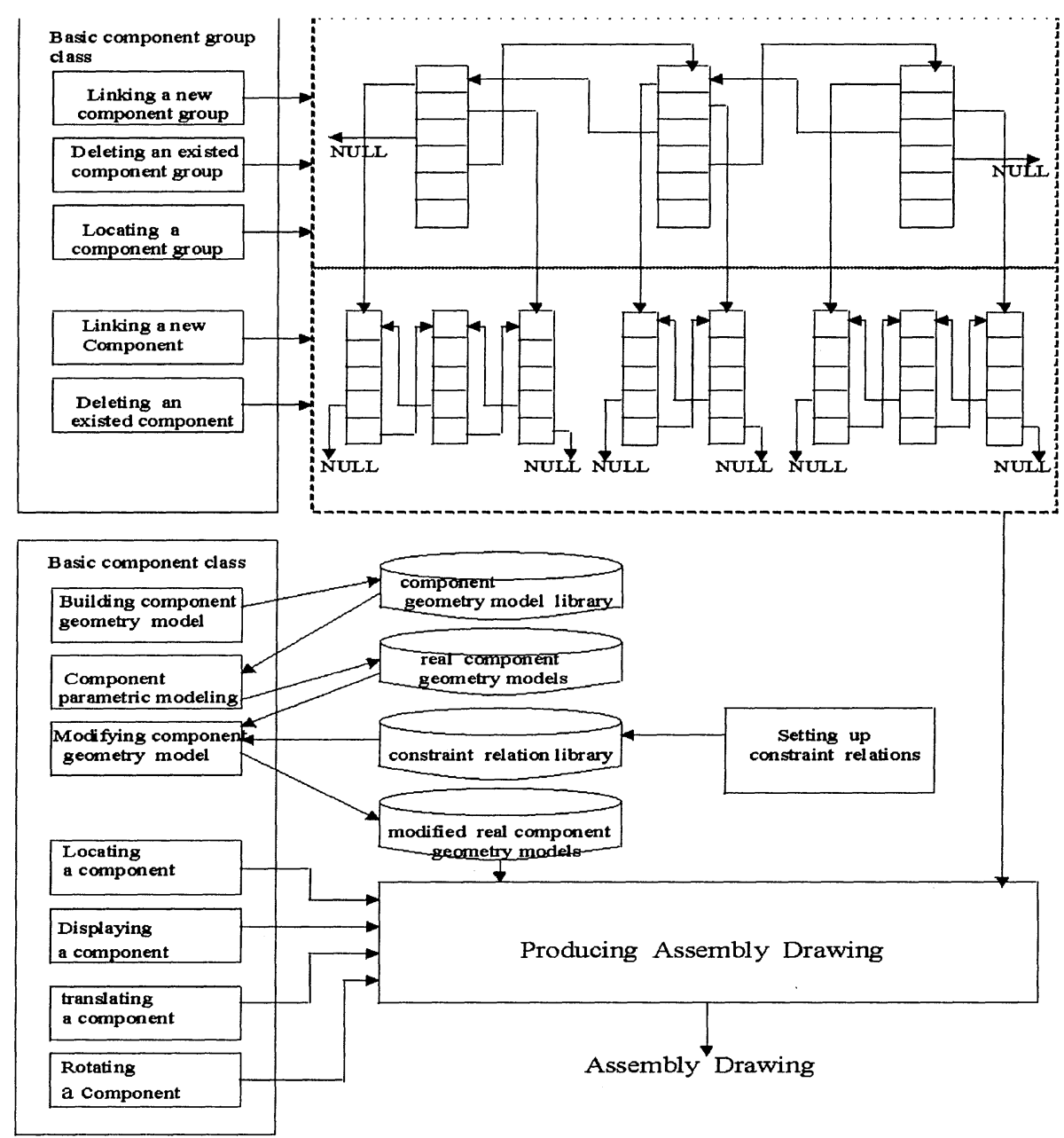

Figure 1 The system architecture principle on objectoriented assembly modeling and design

which can be expressed in variable expressions; A2 stands for the linking relations between components in a component group and those between component groups. A3 stands for constraint relations between two components, so it is a complement to assembling relations. Later we will describe respectively A1, A2, A3.

According to the model described above, the architecture principal of assembly modeling and assembly design system based on object-oriented method is shown figure1. The left-upper box presents the component group class and its operating service programs. The linking relations between component groups and between components in a component group are represented by the double layered and bi- 
directional chained list in right-upper dashed box. The left-down box stands for the component class and its operating service programs. The component geometry model can be built in an interactive or programming way by the module for building component geometry model. The real component geometry models are reproduced by the module for parametric modeling. The assembly constraint relations between components are stored in a constraint relation library, which can be edited by the module for setting up constraint relations. The module for modifying component models generates the final component geometry model by searching for the assembly constraint relations between components. Finally, the module of producing assembly drawing synthesizes an assembly drawing according to the existed component linking relations and the real geometry models. In this way the interacting operations are reduced in order to acquire automatic assembling function.

\section{THE ORIENTED-OBJECTED COMPONENT MODEL IN ASSEMBLY DESIGN}

In the previous formulation, a component model in an assembly is represented by symbol A1. Here every component in the machine can be considered as an object, and any component object should take on three kinds of information [Xiao Xiangzhi,1996]:

(1) Geometry information: including component model's name, and a threedimensional geometry model.

(2) Position information: the coordinates of component base point and the orientation in three-dimension space.

(3) Material information: the component material number

According to the object-oriented design method, the three types of basic information and service programs to manipulate the information should be encapsulated in order to make up the basic component class. The basic component class can be described in $\mathrm{C}++$ as followings:

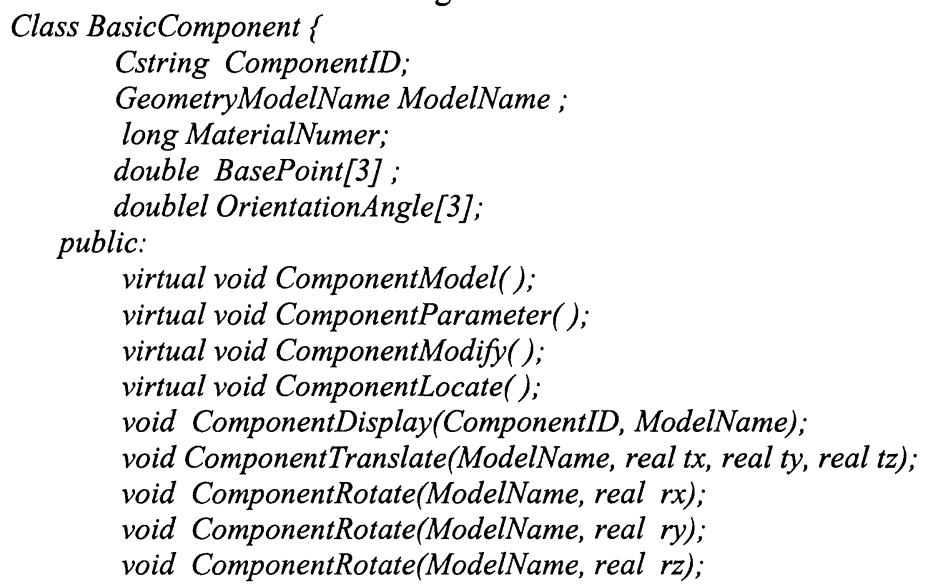


In this class, member function ComponentModel( ), ComponentParameter( ), ComponentModify() and ComponentLocate() are defined as virtual functions which indicate that there may be different methods of geometry modeling, parameter modeling, model modifying and space position determining for different components, so the four functions are to overloaded in sub-class. For example, if a real component model is to be made up, the sub-class expressions based on basic component class will be derived by using inheriting mechanics in object-oriented method.

Except for the above four functions, other functions can be determined fully in basic component class because any component object should take on same displaying, translating, rotating function. These functions are the same for different component objects in various sub-classes, so they are abstracted to set up the basic component class.

\section{THE ESTABLISHMENT OF THE ASSEMBLY MODEL}

The methods described above have defined the component model in assembly modeling. In order to describe the linkage and assembling relations between components in a component group and between component groups, which have been represented as symbol A2, it is discussed below how to build up the assembly relation model.

Here a double-layered and bi-directional chained list, which has been shown in right upper box of figure1, is employed to express the linking and assembling relations. The double-layered and bi-directional chained list is based on following two structures:

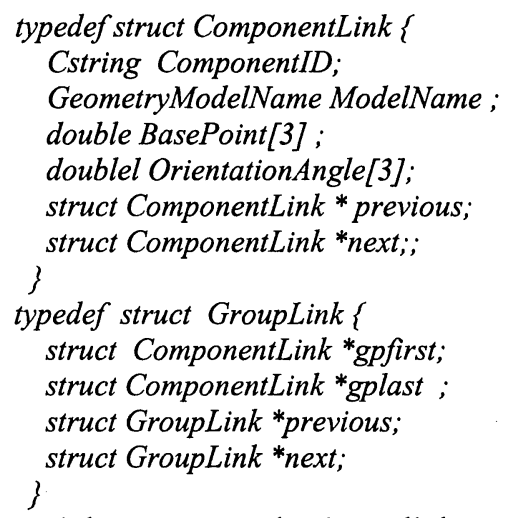

Structure ComponentLink expresses the inter linkage between components in a component group and is considered as the bottom floor of the double layered and bi-directional chained list, while structure GroupLink represents the linkage between the component groups and is thought of as the upper floor of the double layered and bi-directional chained list. From the figure1, it can be seen that the double floor and bi-directional chained list has well represented the linking 
relations in a mechanical device, and it can play an import role in enhancing the efficiency of assembly modeling and design.

In order to set up the oriented-object assembly model, the double floor and bidirectional chained list and its operating service program should be encapsulated in order to make up basic component group class, which is represented in $\mathrm{C}++$ as following:

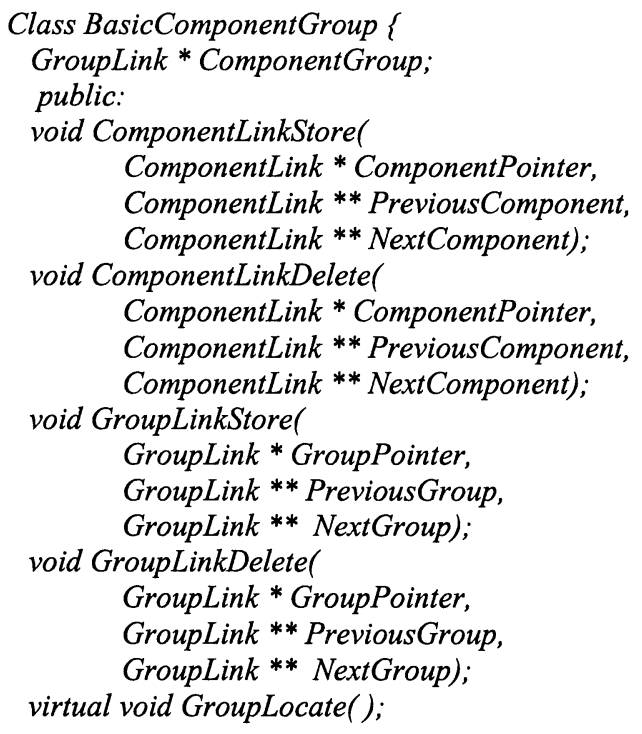

\section{THE SETUP OF THE ASSEMBLY CONSTRAINT MODEL}

An example of constraint relations A3 between components is illustrated in Figure2. By giving the assembly constraint relations, the holes and slot in these components can be generated. 


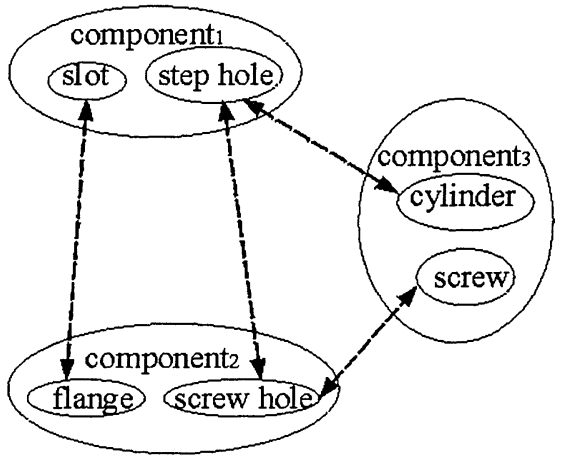

Figure 2 The constrain relations between components

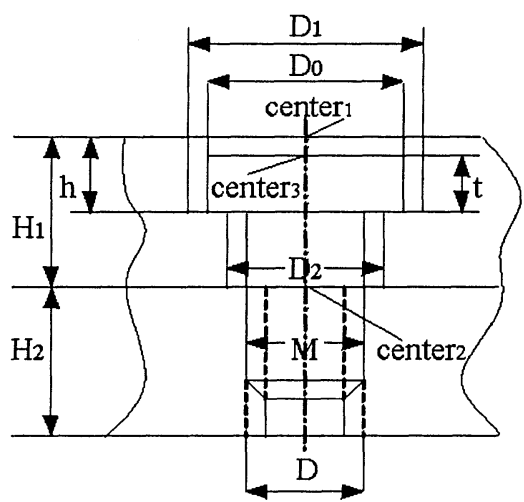

Figure 3 The constrain relations between boards and screw

The constraint relation A3 between components can be described by constraint types and parameters [Wen Jianyong, et al., 1997]. Here, the constraint parameters are expressed as variable expressions, in which the variable types include integer, real, vector and character string. When assembly constraint relations between two components are set up, one side is called as owner, another side is called as connector. When any side is deleted, the relation between two sides will be erased. By the constraint information, the local details of relative components can be designed automatically. For example, the holes and slots shown in figure 2 can be generated by searching for the relations between components. An example of the assembly constraint relations between two boards and a screw is shown in figure3.

As to Figure3, we suppose:

ID1 standing for the upper board

ID2 standing for the bottom board

ID3 standing for the screw

Then the constraint relations between the screw and the upper board and the ones between the screw and the bottom board are described respectively as following:

\section{Relation (screw, upper-board)}

Owner : screw(ID3)

Connector: upper-board(ID1)

\section{Type: Sidestep hole}

\section{Parameter:}

\{

$$
\begin{array}{r}
\text { Center } 1=\{\text { ID1.ComponentLocate }(X), \\
\text { ID1.ComponentLocate }(Y), \\
\text { ID1.ComponentLocate }(Z)\} ; \\
D 1=I D 3 . D 0+2 ; \\
D 2=I D 3 . M+2 ;
\end{array}
$$




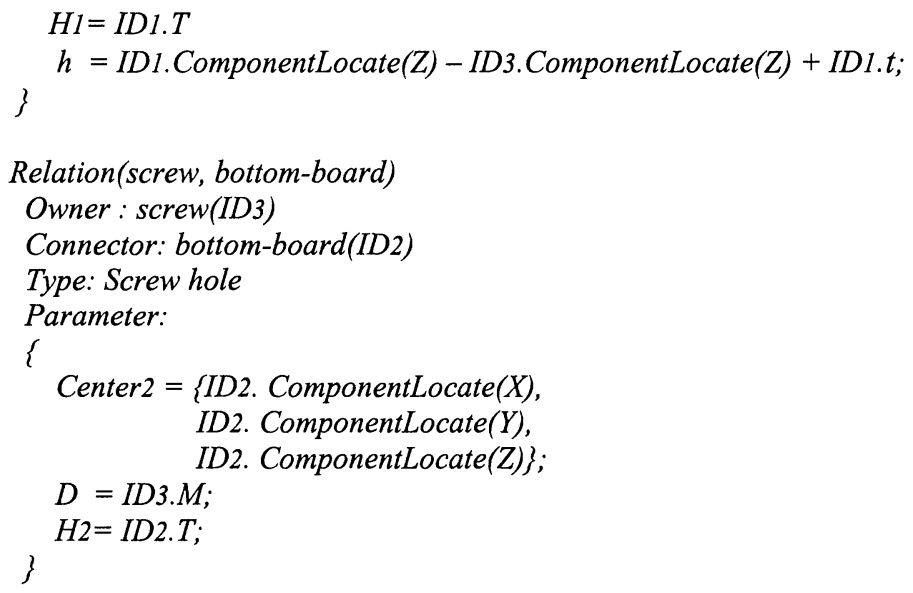

According to the variable expressions of assembly constraint relations, the sidestep hole in the upper board and the screw hole in the bottom board can be generated. In this way, the geometry models of components can be modified by different assembly constraint relations to obtain real component geometry models.

\section{CONCLUSIONS}

The complexity of assembly modeling and CAD system development can be reduced much by using the object-oriented assembly modeling approach presented above. The effectiveness of the introduced assembly modeling approach has been established by the being developed drawing die CAD system and progressive die CAD system.

By defining component groups in the assembly model, the design speed of standard structure can be increased, and standard design can be carried out automatically. By setting up the assembly constraint relations between components, the component's local structure related to assembly can be designed automatically. While the assembly design is finished, the detailed threedimensional component drawing can be also generated automatically.

\section{REFERENCES}

Lee K W and Gossad D C (1985) A Hierarchical Data Structure for Representing Assemblies Part1 2. Computer Aided Design, 1985,(1), 15 24

Mäntylä M.A. (1990) A Modeling system for Top-Down Design of Assembled Product. IBM Journal of Research and Development, 1990, (5), 200 213

Rocheleau D N and Lee K. (1987) System for interactive Assembly modeling. Computer Aided Design, 1987,(2), 65 71 
Shah J J and Rogers M T. (1989) Function Requirement and conceptual Design of the Feature Based modeling. Computer Aided Engineering Journal, 1989, (2), 9 15 Sodhi R and Turner T U. (1994) Towards Modeling of Assembly of Production Design. CAD, 1994, (2), 26 30

Xiao Xiangzhi.(1996) Stamping Process and Dies Computer Aided Design. Chinese National Defense Industry Press, 1996, ISBN7-118-01535-0

Wen Jianyong, et al. (1997) The study of assembly model of the structure CAD system of progressive dies, Chinese Mechanical Engineering, 1997, (8), 16-17

\section{BIOGRAPHY}

Yang Yue gained his Ph.D. in 1992 in the Mechanical Engineering Department of Chongqing University, China. Now he is an associate professor as teacher and researcher of the Mechanical Engineering Department of Changsha Railway University, China. His main research interests are manufacturing system modeling and simulation, manufacturing resource management, computer-aided process planning and $\mathrm{CAD} / \mathrm{CAM}$ integration. At present he is working at the laboratory of design, production and management, University of Twente, Netherlands as a visiting researcher. 